\title{
Facing Big Data Variety in a Model Driven Approach
}

\author{
Marcello Leida, Carlos Ruiz \\ Taiger, Spain \\ Apolonio Morales 13, 28036 Madrid. \\ Email: \{name.surname\}@ taiger.com \\ Paolo Ceravolo \\ Computer Science Department \\ Universita degli Studi di Milano \\ via Bramante 65, 26013 Crema CR. \\ Email: paolo.ceravolo@unimi.it
}

\begin{abstract}
Despite the benefits of investing in Big Data systems are largely recognised, their adoption have been slower than expected. Actually, organisations and companies cannot migrate their systems to new a technological infrastructure without a safe integration to their legacy systems and data. For these reasons, it is required to evolve Big Data technologies with mature functions for supporting portability, interoperability and reusability. This paper illustrates a practical use case exploiting the Model-driven capabilities of the TOREADOR platform as a way to fast track the uptake of business-driven Big Data models.
\end{abstract}

\section{INTRODUCTION}

In the past few years, many organisations in all domains have discovered that, in order to become or remain competitive, they have to deal with business cases where the Volume of data reaches terabytes and even petabytes. The McKinsey Global Institute estimates that data volume is growing $40 \%$ per year, and will grow 44 times larger between 2009 and 2020 [1], similar statements can be applied to the other V-dimensions of the Big Data paradigm (Velocity and Variety). Many IT companies propose to their customers to manage Big Data challenges using a complex stack of technologies [2] including (i) data preparation utilities like Paxata $^{1}$; (ii) a data storage layer, such as Hadoop Distributed File System (HDFS) ${ }^{2}$; (iii) a distributed computing framework such as Hadoop ${ }^{3}$ or Spark $^{4}$; (iv) a data flow layer that applies map-reduce operations to the data partitions of the data storage layer; typically implemented with Big Data specific languages like HiveQL ${ }^{5}$, PigLatin ${ }^{6}$ or $\mathrm{Jaql}^{7}$; and (v) a data query layer implemented with scaling "Not only SQL" databases like Cassandra ${ }^{8}$ or HBase ${ }^{9}$.

\footnotetext{
${ }^{1}$ http://www.paxata.com/

${ }^{2} \mathrm{https} / / /$ hadoop.apache.org/docs/r1.2.1/hdfs_user_guide.html

${ }^{3}$ http://hadoop.apache.org/

${ }^{4} \mathrm{http}: / /$ spark.apache.org/

${ }^{5}$ https://cwiki.apache.org/confluence/display/Hive/LanguageManual

${ }^{6} \mathrm{http}: / /$ pig.apache.org/docs/r0.7.0/piglatin_ref2.html

${ }^{7} \mathrm{http}: / / \mathrm{www} \cdot$ havlena.net/en/programming/jaql-in-hadoop-a-brief-introduction

${ }^{8} \mathrm{http} / / /$ cassandra.apache.org/

${ }^{9}$ https://hbase.apache.org/
}

Despite the benefits of investing in Big Data systems are largely recognised, their adoption have been slow due to a variety of reasons [3]. One of the main reason is that organisations are strongly committed to their systems mainly due to the costs and implications of renewing a technological infrastructure from scratch, therefore their main concern is integrating legacy systems to new technologies, making the best of both worlds. This implies controlling a variety of data formats and interfaces to consistently integrate systems into the Big Data stack. In addition to this scenario, Big Data expertise of the team is usually limited which bring an additional burden to the overall situation. Implementation and deployment of Big Data solutions is therefore an ad-hoc process, crafted around specific requirement and carried out by experienced engineers and developers.

Until now, Big Data application developers have devoted little attention to modelling [4]. However, Model Driven Architectures (MDA) [5] offer a consolidated approach for managing the separation of concerns between components. As it happened with traditional database application in the Seventies, modelling can bring Big Data applications development to adulthood, optimising reuse and reducing development costs, widening the market for Big Data analytics.

The TOREADOR project ${ }^{10}$ is a H2020 project aimed at providing a specification of a fully declarative framework and a model set supporting Big Data Analytics. This paper introduces a typical scenario that can benefit from TOREADOR and discusses the implementation details to be addressed for adopting such a solution. The aim of this paper is describing a study we developed to identify the models, the technologies, the languages, and the interfaces to embody the TOREADOR MDA Big Data platform into a practical use case.

More specifically, the paper is organised as follow: In Section II we frame the general approach adopted, in Section III we illustrate the scenario triggering this work, while in Sections IV we propose a preliminary solution. Finally, in

\footnotetext{
${ }^{10}$ www.TOREADOR-project.eu
} 
Section V we discuss the most relevant challenges we faced addressing this scenario.

\section{Model Driven APPROACH}

International organisations like OMG [6] are specifying new data models aimed at supporting portability, interoperability and reusability through architectural functions. As stated in the introduction, this is a crucial step in the maturing of Big Data architectures to optimise development procedures and widening the adoption of a sound Big Data approach in Data Analytics. Figure 1 illustrates the three core layers of a MDA: the CIM (Computational Independent Model), the PIM (Platform Independent Model) and the PSM (Platform Specific Model):

- The purpose of the Computational Independent Model (CIM) is to describe analytical goals without taking assumptions on the computational implementation.

- The purpose of the Platform Independent Model (PIM) is to describe formally and without ambiguities the procedures to be implemented.

- The purpose of the Platform Specific Model (PSM) is to define the procedures to deploy an infrastructure implementing the procedures.

Translating these layers in a Big Data architecture implies to identify a set of predefined Computation Independent Models (CIM). These models are then mapped into Platform Independent Models (PIM), and according to the the specific deployment constraints, are finally mapped into Platform Specific Models (PSM) and executed in the TOREADOR platform.

\section{SCENARIO}

The following section describes an actual scenario of a big telecommunication company offering provisioning of FiberTo-The-Home (FTTH) connection and related services to residential and commercial units.

\section{Premises:}

- A process instance is created once a customer's order for a service is received (e.g. internet connection with IPTV service). Once the order is received the telecom company starts the service provisioning. This implies to verify that the customer is reached by the fiberoptic cable and in case it is not reached, evaluate the costs and plan for a physical deployment of the cable. If the service requirements are satisfied, a technician needs to physically configure and install the devices in customer's premises. Once all these processes are finally implemented the company starts to provide the service to the customer.

- At the current status, a data warehouse is used to centrally collect all data, spread across several systems, that are describing the overall process. This allows the data analysts to execute Business Intelligence analytics.

\section{Issues:}

- Even if it can appear relatively simple, this process involves different departments, each of them running different sub-processes, storing process execution data into local systems, using identifiers with only local validity. In addition, these departments have different deployments which imply that the data consolidation process needs to be defined for every single use case and department. All these issues result in a situation where the entire process is difficult to follow and monitor for its entire lifespan, not to say to compare it with other executions in order to identify bottlenecks and other problems.

- As described in the premises, in the current situation the process analysts performs analytical tasks using traditional BI tools (Pentaho ${ }^{11}$, Oracle $\mathrm{BI}^{12}$ ). The complexity and intricacies of this widely heterogeneous initial data is mitigated by the data integration step performed using a data warehouse. In order to reduce this complexity, the data warehouse memory is limited to the current snapshot of the process, without possibility to access historical data. This is due to several reasons: data that is spread across multiple systems, modelled according to different domains, usage and policies; in many cases, the status of the process is updated directly into a database table overwriting previous records. This is mostly due to legacy data systems which have been designed to address specific functions and not initially intended for analytical tasks [7]. Moreover, modelling, updating and in general, maintaining a Data Warehouse is a costly task which can not be performed frequently, this will result in data which is not always up-to-date, limiting the possibility of real-time analysis. All these aspects result in an poor knowledge base which offers to the process analyst very limited capabilities.

- Consequently, important analytics, such as for instance failure detection, root cause analysis, proactive problem prediction, process traceability, compliance metrics, and other sort of in-depth analytics, are compromised [8].

Such kind of use case is recurrent in many organisations [7]. Typically, the solutions proposed define an acceptable tradeoff between the cost of providing rich data bases and the cost of maintaining and exploit it. With the advent of Big Data technologies, the trend si that all the data related to a business process execution need to be collected centrally into an unified data representation model. This model need to abstract from domain and system specific data models and it is usually intended as a "unique source of truth" for representing the business process workflows under analysis. Moreover, such execution data needs to be processed, analysed, and visualised quickly and in a timely fashion to gain insights about the execution outcome. Simplifying and covering those common use cases for Big Data is among the aims of the TOREADOR project. The general idea is to exploit a number of declarative and procedural models to define processing pipeline, without the need of dealing with low-level technical details.

\footnotetext{
${ }^{11} \mathrm{http} / / /$ community.pentaho.com/

${ }^{12} \mathrm{https}: / / \mathrm{www}$. oracle.com/solutions/business-analytics/index.html
} 


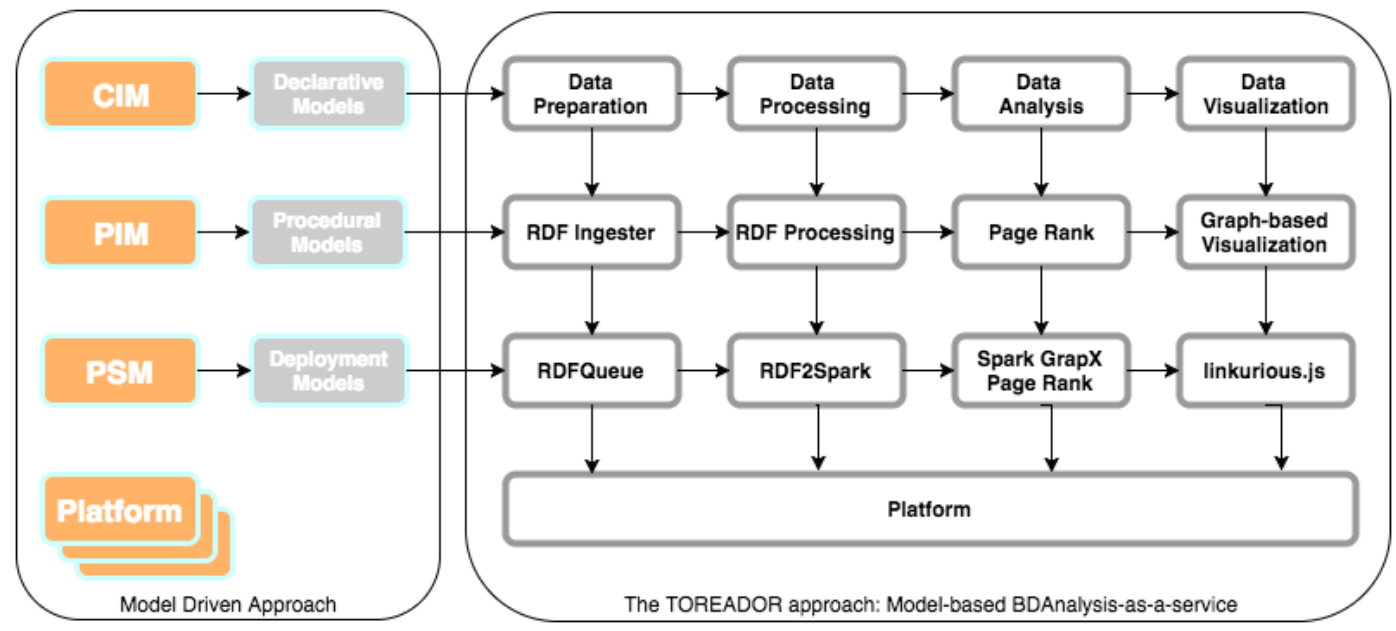

Fig. 1. Model Driven Approach.

\section{APPLYING TOREADOR}

Using the TOREADOR MDA Big Data platform (Figure 1), the analysts can select a number of CIMs, including their parameters, to defined Data Preparation, Processing, Analysis, and Visualisation tasks which are linked to a set of PIMs and PSMs implemented in the TOREADOR platform.

For instance in our scenario, the analysts might define the main building blocks for a processing pipeline, where process execution data is collected centrally into a big data cluster, post-processed in order to recompose and sanitise the process workflow and the dependencies between tasks. In particular:

- Once the process flow has been post-processed, the analytical inspection can start. As an example, the measurement of the PagePank value of every process task can be used to identify critical tasks: assuming that a task with high PageRank is a potential bottleneck and therefore collecting indicators on this tasks may provide valuable information.

- Once indicators are collected a dashboard will be generated to present the identified task and group them around potential issues, as an example: tasks with high variability in execution time, systems or employees that are overloaded and therefore not performing as expected.

The described system can be mapped into the TOREADOR MDA as detailed in the following sections.

\section{A. Implementations Details}

The definition of a common or canonical model is largely recognised as the most safe approach for realising data integration [9]. This definition includes not just the common representation, but also the mapping between each systems integrated in the common model. For example, to integrate the legacy systems of multiple departments we may request that in each system a set of probes are deployed. These probes "listen" for process execution data and convert this data into the format imposed by the common model. In the use case we present, the data model chosen is the Resource Description
Framework (RDF) [10] a W3C standard used as a general purpose language for representing information in the Web. The RDF data model is organised in triples, i.e. predicates relating one object to another, where objects are any web resource identified by an URI. The RDF model is extended according to the vocabulary described in [11] which provides a basic business process model schema supporting analytical processes, as exploited in [12], [13], [14].

The implications of adopting a centralised layer for data storage are not limited to data normalisation. Indeed, systems that support data processing transparently to data creation and analysis offer a real-time representation of process execution [15]. This is achieved implementing probes as data streams emitters, which constantly update the centralised layer with new data, converted into an RDF representation and sent to a message queue. In our scenario, the triples contained in the data layer are analysed by an RDF to Spark conversion library and processed to calculate the PageRank of each task. The result is then visualised using a Javascript library and presented to the analyst; the workflow characterising this process is illustrated in Figure 2.

The described components can be mapped easily into the TOREADOR MDA: at first step the analyst will define the processing pipeline as a set of CIM models: in order to help the TOREADOR platform in the selection of the correct PIM and PSM, a set of additional parameters are required from the analyst to complete the CIM models; as an example, in this specific case, the analysts will indicate the data representation model to use and the fact of monitoring streams of data (usage of a message queue). This information is enough to select the correct PSM. Then the analysts will specify the type of analysis (e.g. by selecting a particular CIM model), which depending on the storage and processing system adopted will be mapped to a specific PSM.

Finally the results of the analysis are presented visually with a CIM Data Visualisation Model selected by the analyst among the different models provided by the TOREADOR systems 


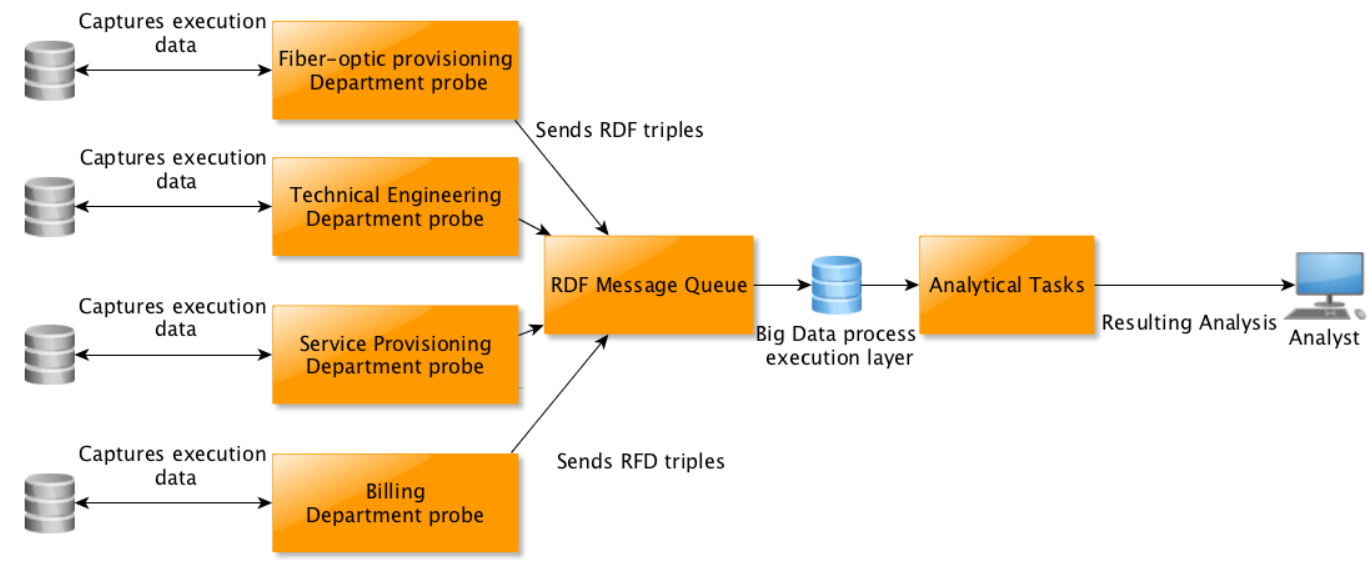

Fig. 2. The data flow implemented in the proposed scenario.

based on the parameters and the deployment environment (e.g. web application).

In detail, with regards to the Figure 1, the Data Preparation task at CIM level requires the analyst to identify the data elements to be stored to perform process analytics. These elements are monitored by an RDF ingester responsible for collecting, curating, and storing data. The definition of the Data Processing task to be implemented is defined at the CIM level by listing the components that must be involved in the processing stage, for example the fact of monitoring streams of data from RDF queues will require an RDF translator, a message queue and a processing component (e.g. a RDF2Spark component to process and store RDF triples in a distributed system such as Spark). At the PIM level the process execution information is translated in a set of RDF triples and submitted to a message queue. Note that the general structure of these modules can be derived by the list of components defined at CIM level. Except for the concrete localisation of the components deployed, this information is enough to select the correct PSM: an RDF translator, i.e. a RDF2Spark component to process and store RDF triples in a distributed system such as Spark. The definition of the Data Analytics task to be implemented is defined at the CIM level by simply referring to one of the analytics types included in the TOREADOR library. This will limit the options that the analyst can specify at the PIM level where she has to select the algorithm to be implemented (e.g. PageRank). Depending on the storage and processing system adopted (Spark) this algorithm is mapped by the TOREADOR platfom to a specific PSM. For example, an implementation of the PageRank algorithm using GraphX library ${ }^{13}$. Data Visualization is the last stage, where the results of the selected algorithm are presented visually. Since RDF data and process execution workflows can be seen as a particular case of graphs, the system will select a Graphbased visualisation library as PIM, which depending on the deployment environment (e.g. web application) will select the

\footnotetext{
${ }^{13} \mathrm{http}: / /$ spark.apache.org/graphx/
}

correct PSM for the visualisation step (e.g. use of Linkurious javascript library ${ }^{14}$ ).

\section{Adressed Challenges}

In this section we present some important aspects that must be considered when dealing with RDF data in a Big Data processing environment and represents the lessons learned from the investigation of this scenario.

\section{A. Process Identification}

Entity identification and reconciliation [16] is critical when trying to establish accurate relationships between entities from data sources subject to different life-cycles. This is due to the fact that different manifestations of the same entity may vary their identify conditions in different ways within the different data sources. The problem is particularly difficult when the analytics insist on processes, as data sources may provide identifiers for events and entities involved in a process but not for the process itself.

The solution we propose is based on the dynamic definition of the identify criteria of a process. One of the probes sending data to the RDF queue is responsible for generating the identity criteria of a process by exposing an Entity Resolution service. The other probes identify a process based on the criteria defined by the Entity Resolution service. In case this criteria change probes will react accordingly.

For example the commercial department receives an order and starts sending the triples concerning the execution of the Order Preparation task. This also implies the definition of all the attributes that will identify this process (order number, customer name, request, telephone number). Then the technical department receives the order that an engineer must go to a specific address for installation and configuration of the required devices. When the listener receives triples on the queue it will use the attributes exposed by the Entity Resolution service to aggregate the triples that belong to the

\footnotetext{
${ }^{14} \mathrm{http} / / /$ linkurio.us/toolkit/
} 
Listing 1. The GraphX Property Graph

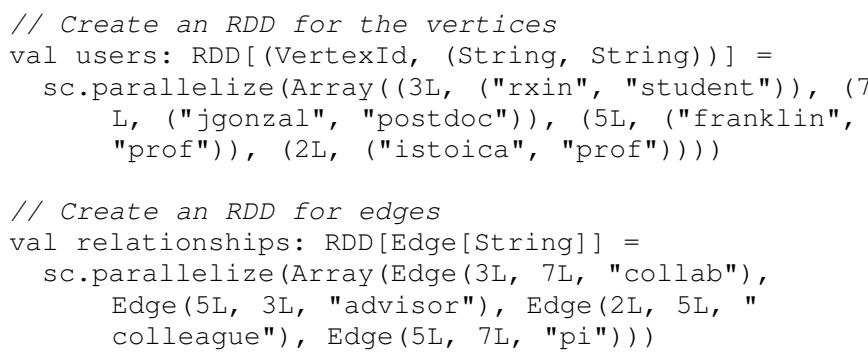

same process, and, if necessary, aggregating the attributes that have the same value all over the process into a new instance.

\section{B. Data Interoperability in Spark}

Apache Spark provides programmers with an application programming interface for interacting with distributed memory organised according to a data structure called a Resilient Distributed Dataset (RDD), implementing a fault-tolerant distribution across a computing cluster. Spark provides higherorder functions that facilitate the implementation of iterative algorithms (for example PageRank) that are required to update the dataset in the distributed memory multiples times. This kind of tasks generally don't work well on MapReduce implementations that force a linear data flow. Moreover, the latency of Spark is reduced compared to traditional MapReduce implementations because the memory management approach implemented avoid all the disk I/O that is typical required by linear MapReduce flows.

GraphX is a graph library that runs on top of Spark [17]. The graph data structure used by GraphX is a combination of an RDD for vertices and one for edges. At first sight, the RDF triples could be mapped storing objects in the RDD vertices and predicates in the RDD edges. However the GraphX vertices and edges are created using ternary operators, where in vertices the first argument is used as the node identifier while in edges the two first arguments are used as identifiers for the origin and target nodes, as illustrated in Listing 1. Very litter space is left for literal properties, i.e. properties associated to literal values such as strings and integers, and extra information such as context, i.e. annotating the context in which a resource is generated [18]. The first limitation may be addressed by adding vertices for describing literal values and edges for properties. The second limitation is not straightforward as it is discussed in next section.

\section{The Contextualisation Problem}

The contextualisation problem of distributed data structures is related to the fact that the referential integrity of a statement may be safe at the generation time but invalidated when integrating this statement with others generated in a different context, i.e. different sources, different stages [18]. For instance, in Listing 2, when context is not considered, the integration of $s 2$ and $s 3$ merge the information of nodes host losing their original values: different statements about the
Listing 2. Integrating statements from multiple sources

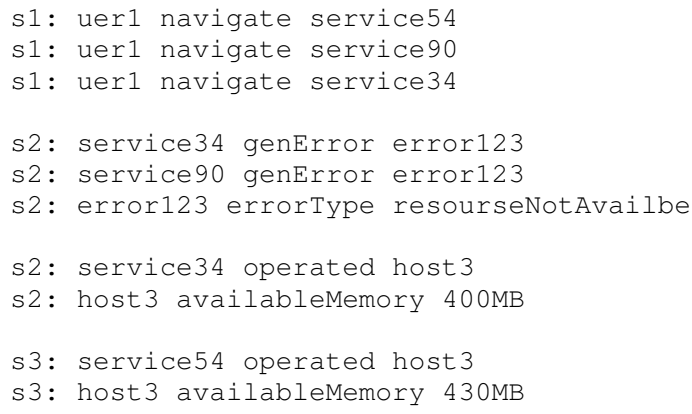

availableMemory was generated in different conditions but considering the statements by themselves we cannot identify their original value. Note that, the integration of $s 1$ and $s 2$ maintains the statements valid only because the property of the nodes error is invariant at the different contexts. In the literature this problem is adressed by attaching statements to a descriptor for their context [19]. This way two predicates or properties of the same type can be distinguished if generated in different contexts: $s 2$ : availableMemory is different from s3 : availableMemory.

A consistent mapping of RDF statements in GraphX implies the definition of contextual descriptors for predicates and properties. This aim could be for instance achieved by introducing custom schemata ${ }^{15}$ to prefix the URIs used for naming resources or by using the authentication section typically not exploited by applications.

\section{CONCLUSIONS}

In this work, we provided a description of the the models, the technologies, the languages, and the interfaces to embody the TOREADOR MDA Big Data platform into a practical use case. The use case presented is a FTTH service delivery process of a big telecommunication company, which has to deal with legacy systems and process data spread across different department. The paper presented a processing workflow and how this flow can be deployed into the TOREADOR platform, following MDA principles. The TOREADOR project is aimed at exploring further scenarios to then define a modelbased, platform-independent Big Data processing and analytics framework.

\section{ACKNOWLEDGEMENT}

This project has received funding from the European Union's Horizon 2020 research and innovation programme under the TOREADOR project, grant agreement No 688797.

\section{REFERENCES}

[1] Oracle, "Oracle: Big data for the enterprise," 2014 [Online]. Available: http://www.oracle.com/us/products/database/ big-data-for-enterprise-519135.pdf

\footnotetext{
${ }^{15}$ While most URI schemata were originally designed to be used with a particular protocol, the current standard does not impose this condition: prefix not associated with any specific protocol are available for example file or news.
} 
[2] V. Borkar, M. J. Carey, and C. Li, "Inside big data management: ogres, onions, or parfaits?" in Proceedings of the 15th International Conference on Extending Database Technology. ACM, 2012, pp. 3-14.

[3] R. Malcolm, C. Morrison, T. Grandison, S. Thorpe, K. Christie, A. Wallace, D. Green, J. Jarrett, and A. Campbell, "Increasing the accessibility to big data systems via a common services api," in Big Data (Big Data), 2014 IEEE International Conference on. IEEE, 2014, pp. 883-892.

[4] K. Slavakis, G. B. Giannakis, and G. Mateos, "Modeling and optimization for big data analytics: (statistical) learning tools for our era of data deluge," IEEE Signal Processing Magazine, vol. 31, no. 5, pp. 18-31, Sept 2014.

[5] "Model driven architecture." [Online]. Available: http://www.omg.org/ mda/

[6] "Object management group." [Online]. Available: http://www.omg.org

[7] P. Taylor, M. Leida, and B. Majeed, Data-Driven Process Discovery and Analysis: First International Symposium, SIMPDA 2011, Campione d'Italia, Italy, June 29 - July 1, 2011, Revised Selected Papers. Berlin, Heidelberg: Springer Berlin Heidelberg, 2012, ch. Case Study in Process Mining in a Multinational Enterprise, pp. 134-153. [Online]. Available: http://dx.doi.org/10.1007/978-3-642-34044-4_8

[8] W. van der Aalst, A. Adriansyah, A. K. A. de Medeiros, F. Arcieri, T. Baier, T. Blickle, J. C. Bose, P. van den Brand, R. Brandtjen, J. Buijs, A. Burattin, J. Carmona, M. Castellanos, J. Claes, J. Cook, N. Costantini, F. Curbera, E. Damiani, M. de Leoni, P. Delias, B. van Dongen, M. Dumas, S. Dustdar, D. Fahland, D. R. Ferreira, W. Gaaloul, F. van Geffen, S. Goel, C. Gunther, A. Guzzo, P. Harmon, A. ter Hofstede, J. Hoogland, J. E. Ingvaldsen, K. Kato, R. Kuhn, A. Kumar, M. La Rosa, F. Maggi, D. Malerba, R. Mans, A. Manuel, M. McCreesh, P. Mello, J. Mendling, M. Montali, H. M. Nezhad, M. zur Muehlen, J. Munoz-Gama, L. Pontieri, J. Ribeiro, A. Rozinat, H. S. Perez, R. S. Perez, M. Sepulveda, J. Sinur, P. Soffer, M. Song, A. Sperduti, G. Stilo, C. Stoel, K. Swenson, M. Talamo, W. Tan, C. Turner, J. Vanthienen, G. Varvaressos, E. Verbeek, M. Verdonk, R. Vigo, J. Wang, B. Weber, M. Weidlich, T. Weijters, L. Wen, M. Westergaard, and M. Wynn, "Process mining manifesto," in Lecture Notes in Business Information Processing, F. Daniel, K. Barkaoui, and S. Dustdar, Eds., vol. 99, no. 2. Springer, 2012, pp. 169-194. [Online]. Available: http://dx.doi.org/10.1007/978-3-642-28108-2 \_19

[9] A. Calì, D. Calvanese, G. De Giacomo, and M. Lenzerini, "Data integration under integrity constraints," in Seminal Contributions to Information Systems Engineering. Springer, 2013, pp. 335-352.

[10] RDF/XML Syntax Specification, World Wide Web Consourtium, February 2004. [Online]. Available: http://www.w3.org/TR/2004/ REC-rdf-syntax-grammar-20040210/

[11] M. Leida, B. Majeed, M. Colombo, and A. Chu, Data-Driven Process Discovery and Analysis: Second IFIP WG 2.6, 2.12 International Symposium, SIMPDA 2012, Campione d'Italia, Italy, June 18-20, 2012, Revised Selected Papers. Berlin, Heidelberg: Springer Berlin Heidelberg, 2013, ch. A Lightweight RDF Data Model for Business Process Analysis, pp. 1-23. [Online]. Available: http://dx.doi.org/10.1007/978-3-642-40919-6 \_1

[12] S. Thalmann, M. Manhart, P. Ceravolo, and A. Azzini, "An integrated risk management framework: measuring the success of organizational knowledge protection," International Journal of Knowledge Management (IJKM), vol. 10, no. 2, pp. 28-42, 2014.

[13] P. Ceravolo and F. Zavatarelli, "Knowledge acquisition in process intelligence," in Information and Communication Technology Research (ICTRC), 2015 International Conference on. IEEE, 2015, pp. 218-221.

[14] F. Arigliano, A. Azzini, C. Braghin, A. Caforio, P. Ceravolo, E. Damiani, V. Savarino, C. Vicari, and F. Zavatarelli, "Knowledge and business intelligence technologies in cross-enterprise environments for italian advanced mechanical industry." in SIMPDA, 2013, pp. 104-110.

[15] M. Leida, A. Chu, M. Colombo, and B. Majeed, "Extendible data model for real-time business process analysis," in Industrial Engineering and Engineering Management (IEEM), 2012 IEEE International Conference on. IEEE, 2012, pp. 1593-1597.

[16] L. Getoor and A. Machanavajjhala, "Entity resolution: theory, practice \& open challenges," Proceedings of the VLDB Endowment, vol. 5, no. 12, pp. 2018-2019, 2012.

[17] J. E. Gonzalez, R. S. Xin, A. Dave, D. Crankshaw, M. J. Franklin, and I. Stoica, "Graphx: Graph processing in a distributed dataflow framework," in 11th USENIX Symposium on Operating Systems Design and Implementation (OSDI 14), 2014, pp. 599-613.
[18] P. Bouquet, L. Serafini, and H. Stoermer, "Introducing context into rdf knowledge bases." in SWAP, vol. 5, 2005, pp. 14-16.

[19] J. Bao, J. Tao, D. L. McGuinness, and P. Smart, "Context representation for the semantic web," 2010 . 